\title{
Megatrials in type 2 diabetes. From excitement to frustration?
}

\author{
S. Del Prato
}

Published online: 17 April 2009

(C) Springer-Verlag 2009

\begin{abstract}
Whether glycaemic control may result in a reduction of cardiovascular $(\mathrm{CV})$ risk has been a matter of continuous discussion and investigation. Epidemiological analyses have extensively suggested a relationship between glycaemic control and CV events; however, the results of intervention trials have been less conclusive. The UKPDS reported a $16 \%$ reduction in the risk of myocardial infarction, but this reduction was not statistically significant. The results of the Kumamoto and PROactive studies could not allow any firm conclusions to be drawn either, because of limited size and the defined primary endpoint, respectively. The results of the Action to Control Cardiovascular Risk in Diabetes (ACCORD) and Action in Diabetes and Vascular Disease: Preterax and Diamicron Modified Release Controlled Evaluation (ADVANCE) trials and the Veteran Administration Diabetes Trial (VADT) were published in rapid succession over the second half of 2008 and at the beginning of 2009. A total number of almost 25,000 type 2 diabetic patients were recruited in these three trials, which assessed the effect of intensive glycaemic control vs conventional treatment on well-defined CV endpoints. In spite of the achievement and maintenance of strict glycaemic control $\left(\mathrm{HbA}_{1 \mathrm{c}}<7.0 \%\right)$, no beneficial effect of intensive therapy was apparent in any of the studies. At the same time these results were presented, the results of an analysis of the 10 year follow-up of the UKPDS also became available and provided a more optimistic view of the potential benefit of achieving good
\end{abstract}

S. Del Prato $(\bowtie)$

Department of Endocrinology and Metabolism,

Section of Diabetes and Metabolic Diseases, Ospedale Cisanello,

Via Paradisa 2,

56126 Pisa, Italy

e-mail: delprato@immr.med.unipi.it

glycaemic control. The relative risk reductions for myocardial infarction and all-cause mortality were significantly lower in the patients who initially received the intensive treatment compared with those in the conventional treatment arm. Moreover, the initial benefit in terms of microvascular complications observed at the end of the intervention trial remained unaltered at follow-up. Once again the debate on the importance of glycaemic control in preventing macrovascular complications remains unsettled. These results, however, require some reconciliation, and the objective of this commentary is to analyse a series of elements, including the changes in the treatment approach to $\mathrm{CV}$ risk factors in type 2 diabetes, the effect of glucoselowering agents, and the characteristics of the patients included in the different trials, that should be taken into account when interpreting the results of intervention trials in type 2 diabetes.

Keywords Cardiovascular disease - Clinical trials ·

Diabetic complications · Disease duration .

Macrovascular complications · Microvascular complications - Risk of hypoglycaemia - Type 2 diabetes

Type 2 diabetes treatment

\section{Abbreviations}

ACCORD Action to Control Cardiovascular Risk in Diabetes

ADVANCE Action in Diabetes and Vascular Disease: Preterax and Diamicron Modified Release Controlled Evaluation

ADA

CAC

$\mathrm{CV}$

UGDP

VADT
American Diabetes Association Coronary artery calcium Cardiovascular University Group Diabetes Program Veteran Administration Diabetes Trial 


\section{In the beginning...the UKPDS...}

The expectations in Barcelona were high 10 years ago when, at the 34th Annual Meeting of the EASD, a large audience anxiously awaited the results of the first large long-term intervention trial in type 2 diabetes following the debacle of the University Group Diabetes Program (UGDP) [1]. The results of the UKPDS were welcomed as they showed that intensive treatment, as opposed to the conventional approach, was associated with a significant reduction in diabetes-related events [2]. This excitement soon translated into official recommendations from the major scientific and professional organisations. The American Diabetes Association (ADA), for instance, emphatically stated that 'the results of the UKPDS mandate that treatment of type 2 diabetes includes aggressive efforts to lower blood glucose levels as close to normal as possible' [3].

Yet, some questions remained unanswered. While the effect of glycaemic control on microvascular complications was fully apparent, its effect on macrovascular disease was less clear. In spite of a sizable $16 \%$ reduction in the risk of myocardial infarction, borderline statistical significance was achieved, with a $p$ value of 0.052 [2]. No third decimal place in the history of medicine has stimulated such an amount of discussion and speculation. The publication of the results of the Kumamoto study [4] did not help much. In this small $(n=110)$ trial, the administration of intensive insulin treatment to Japanese type 2 diabetic patients was associated with a significant reduction in microvascular complications. After the initial 8 year follow up, the number of cardiovascular (CV) events was $\sim 50 \%$ lower in the intensive group than in conventionally treated individuals, but the absolute number of events was too small to allow a formal statistical analysis, and no conclusive results were obtained. ...then, the PROactive...

It was a few years later, in Athens, that thousands of diabetologists eagerly anticipated the announcement of the results of the PROactive study [5]. The addition of pioglitazone to the existing glucose-lowering therapy of type 2 diabetic patients at high $\mathrm{CV}$ risk improved $\mathrm{HbA}_{1 \mathrm{c}}$ in a significant manner, but produced controversial results as regards CV events. In spite of significant improvement of glycaemic control, the primary endpoint (a composite of $\mathrm{CV}$ events, mortality and procedures) was not affected, while a significant difference was achieved in pre-specified secondary endpoints [5]. To cut a long story short, the question of whether strict glycaemic control could improve $\mathrm{CV}$ outcome in type 2 diabetes had still not been answered.

\section{...and now, the Megatrials}

The results of the Action to Control Cardiovascular Risk in Diabetes (ACCORD) [6], Action in Diabetes and Vascular Disease: Preterax and Diamicron Modified Release Controlled Evaluation (ADVANCE) [7] and Veteran Administration Diabetes Trial (VADT) [8] were published in rapid succession in 2008 and the beginning of 2009. A total of almost 25,000 type 2 diabetic patients have been enrolled in these trials, which were designed to determine whether achieving and maintaining normal or near-normal plasma glucose levels could reduce the risk of CV events and CV mortality (Table 1). The results are known, the disappointment still tangible- no significant reduction of $\mathrm{CV}$ risk was apparent. Even worse, the ACCORD trial was prematurely interrupted because of excess mortality among intensively treated patients [6]. The VADT results on microvascular outcome are even more incredible. Of the multiple aspects of retinopathy, nephropathy and neuropathy assessed in the trial, the only statistically

Table 1 Summary of the main features and results of the most recent intervention trials in type 2 diabetic patients

\begin{tabular}{llcc}
\hline Variable & VADT $(n=1,700)$ & ACCORD $(n=10,250)$ & ADVANCE $(n=11,140)$ \\
\hline HbA $_{1 \mathrm{c}}(\%)^{\mathrm{a}}$ & 8.4 vs 6.9 & 7.5 vs 6.4 & 7.3 vs 6.5 \\
Primary outcome & $\begin{array}{c}\text { MI, stroke, death from CV causes, new or } \\
\text { worsening CHF, revascularisation }\end{array}$ & $\begin{array}{c}\text { Non-fatal MI, non-fatal } \\
\text { stroke, CVD death }\end{array}$ & $\begin{array}{c}\text { Non-fatal MI, non-fatal } \\
\text { stroke, CVD death }\end{array}$ \\
& $\begin{array}{l}\text { and inoperable CAD, amputation } \\
\text { for ischaemic gangrene }\end{array}$ & $0.90(0.78-1.04)$ & $0.94(0.84-1.06)$ \\
HR $(95 \% \mathrm{CI})$ for primary outcome & $0.87(0.730-1.04)$ & $1.22(1.01-1.46)^{\mathrm{b}}$ & $0.93(0.83-1.06)$ \\
HR $(95 \% \mathrm{CI})$ for mortality & $1.065(0.801-1.416)$ & & \\
\hline
\end{tabular}

\footnotetext{
${ }^{\mathrm{a}}$ Conventional vs intensive

${ }^{\mathrm{b}} p=0.04$

CAD, coronary artery disease; $\mathrm{CHF}$, congestive heart failure; CVD, cardiovascular disease; MI, myocardial infarction
} 
significant effect of strict glycaemic control was a decrease in the conversion from normo- to micro- or macroalbuminuria [8]. These results seem to be at variance with the significant reduction in the relative risk of microvascular complications reported in the ADVANCE trial [7]. However, a closer look at these results reveals that the risk reduction is entirely accounted for by a decreased incidence of albuminuriareflecting the finding of the VADT.

Can we draw any conclusions from the results of these intervention trials? Do they undermine the value of good glycaemic control in preventing or slowing progression of diabetic complications? To address these questions a number of issues need to be considered.

\section{Improved care for $\mathrm{CV}$ risk in diabetes}

The therapeutic approach to type 2 diabetes has greatly changed since the results of the UKPDS were reported in 1998. The use of statins and newly developed agents for hypertension, more liberal use of aspirin and smoking cessation are all increasingly becoming part of the standard approach. This is well reflected in the three most recent trials. More than $86 \%$ of the ACCORD [6] and VADT [8] study populations were on statins. This percentage $(\sim 46 \%)$ was lower among the ADVANCE [7] study participants, but a large proportion of this study population was from Asia, making a direct comparison with ACCORD and VADT difficult. Aspirin was prescribed for $\sim 94 \%, 76 \%$ and $57 \%$ of the populations of the three trials, respectively [6-8]. Average blood pressure was $130 / 70 \mathrm{mmHg}$ in the ACCORD trial and the VADT, and $130 / 75 \mathrm{mmHg}$ in the ADVANCE trial. The proportion of smokers at the end of the three trials was $\leq 10 \%$. None of the trials showed a difference between patients on intensive and conventional treatment, reducing the likelihood of a substantial impact of tight glycaemic control. The multifactorial treatment strategy used in the intervention trials is not simply the result of careful study design, but reflects a positive trend in the general therapeutic approach of type 2 diabetes. The recent analysis of the US National Health and Nutrition Examination Survey 1999-2004 [9] reported that, over the 5 year period, there was an increase in the percentage of type 2 diabetic patients achieving their target for $\mathrm{HbA}_{1 \mathrm{c}}(57 \%$; $+21 \%)$, blood pressure $(48 \% ;+12 \%)$ and total cholesterol $(54 \% ;+6 \%)$. Although the proportion of individuals at target for all three variables was low (13.2\%), this was a $100 \%$ improvement on the percentage 5 years earlier. The picture emerging from these trials and from general practice is in accordance with the steady decline in CV mortality in the diabetic population [10] and account for the finding of lower mortality rates than predicted in the three trials. In summary, the results of the recent trials provide a clue as to the explanation for the more favourable overall outcome in diabetic patients in recent years. Under these conditions the potential absolute risk reduction attributable to glucose lowering is likely to be difficult to demonstrate, requiring an even larger number of patients or much longer follow-up.

Multifactorial intervention may also exert an effect on microvascular complications. This possibility becomes apparent if we switch for a moment to type 1 diabetes, a condition in which hyperglycaemia is considered the main pathogenetic factor for micro- and macrovascular complications [11]. Data on the Italian cohort of the EURODIAB population gave us the opportunity to analyse the predictive factors for prevalent and incident microvascular complications (retinopathy and nephropathy). Multivariate analysis revealed not only glucose control and disease duration as independent predictors of the development of microvascular complications, but also dyslipidaemia, blood pressure and even a derived estimation of insulin sensitivity. The role of these factors has been indicated by several observations $[12,13]$ and confirmed in intervention trials. In the UKPDS a significant interaction between $\mathrm{HbA}_{1 \mathrm{c}}$ and blood pressure was been observed with respect to both micro- and macrovascular complications and mortality [14]. The results of the Steno-2 Study are even more suggestive, since multifactorial treatment reduced the risk of $\mathrm{CV}$ mortality by $53 \%$, and was also associated with a risk reduction of $61 \%$ for nephropathy, $58 \%$ for retinopathy and $63 \%$ for neuropathy [15].

Thus, the results of the most recent trials, when analysed together with data from previous studies and with the available epidemiological evidence, rather than stimulating new discussion on the relative role of each risk factor, support the need for a comprehensive treat-to-target approach for all of them.

\section{Different glucose medications, different outcomes?}

The possibility that, irrespective of the extent of glycaemic control they can provide, the different pharmacological agents have varying effects on $\mathrm{CV}$ outcome has been repeatedly suggested, but there is currently no conclusive evidence of this. The UGDP [1] was stopped because of an apparent increased mortality rate with sulfonylureas and the results were dismissed because of statistical biases [16]. No harm was observed with sulfonylureas in the UKPDS [2], although metformin treatment was associated with better CV outcome [17], a benefit lost with the combination of the two medications [2]. These results are in agreement with large retrospective analyses. In the survey of Evans et al. [18], in spite of similar glycaemic control $\left(\mathrm{HbA}_{1 \mathrm{c}} \sim 8 \%\right)$, the mortality rate was lower for metformin than for sulfonylur- 
eas, with the combination of the two producing an intermediate rate. Among the older adults of the Cardiovascular Health Study [19], a greater mortality rate was observed for those treated with insulin compared with those on oral hypoglycaemic agents, even though there was no apparent difference between these two subgroups in terms of fasting plasma glucose $(\sim 10 \mathrm{mmol} / \mathrm{l})$. In the Euro Heart Survey, in diabetic patients with coronary artery disease, insulin was related to a more serious prognosis than were oral glucose-lowering medications [20]. Interestingly, in the VADT, insulin therapy at baseline was an independent predictor of subsequent CV mortality [8]. Whether this association reflects a true medication-related risk, or, rather, different severity of the disease condition remains a matter of debate, but it is intriguing that agents that may cause hypoglycaemia are more often associated with increased $\mathrm{CV}$ risk.

\section{Hypoglycaemia: a trigger for CV events?}

In the VADT [21], at baseline, at least $50 \%$ of the patients were already on insulin, and $70 \%$ of the study population were taking metformin. A much lower proportion of patients were taking rosiglitazone $(<15 \%)$, and the percentage taking sulfonylureas was almost insignificant $(<3 \%)$. After randomisation, the number of patients on insulin increased in both the intensive and the conventional arms, reaching $85 \%$ and $75 \%$, respectively, at the end of the fourth year (Fig. 1). The use of glimepiride increased dramatically in both arms $(57 \%$ vs $42 \%$ at year 4), although the increase was greater in intensively treated patients. The increased use of glucoselowering agents was associated with an increase of $117 \%$ in the incidence of severe hypoglycaemic events [8].
A similar picture emerges from the ACCORD study, in which intensive treatment was associated with a significantly greater rate of severe hypoglycaemic events [6]. In both the ACCORD study [22] and the VADT a clear association between severe hypoglycaemia and CV events was found, although no clear-cut cause-effect relationship could be proven [8].

It should be noted that the absolute rate of severe hypoglycaemia remains lower than the rates reported in trials performed in type 1 diabetic patients $[2,6-8,11]$, but hypoglycaemia may still be a matter of concern in older type 2 diabetic patients, particularly in those with previous CV events. Hypoglycaemia can be associated with (1) activation of adrenergic response [23], (2) impaired flexibility in substrate shift in the diabetic myocardium [24], (3) QTc prolongation and cardiac rate/rhythm disturbances [25], and (4) excessive glucose fluctuations with marked activation of oxidative stress [26]. A further potential indicator of patient vulnerability is represented by diabetic autonomic neuropathy. In the VADT [8], 43\% of the patients had diabetic neuropathy at study entry. Although not specified, such a high prevalence probably includes patients with autonomic neuropathy, a condition associated, in an independent manner, with a greater rate of CV mortality [27].

\section{Glucose control-the longer the disease duration, the smaller the benefit?}

The likely presence of autonomic neuropathy in a certain proportion of the VADT participants bring us to focus on the features of the type 2 diabetic patients enrolled in this and the other major intervention trials. As mentioned
Fig. 1 Proportion of patients receiving the glucose-lowering treatments insulin (a), glimepiride (b), rosiglitazone (c) and metformin (d) at study entry (baseline) and during the initial 4 years of the VADT [21] Darker blue columns, conventional treatment; lighter blue columns, intensive treatment
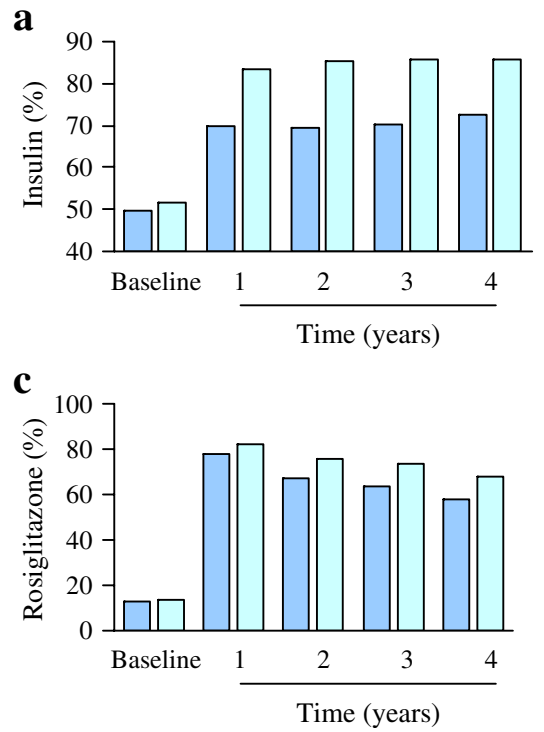

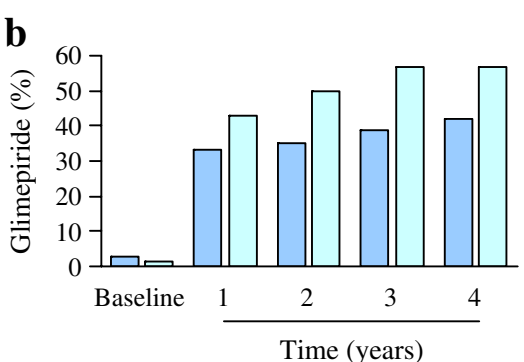

d

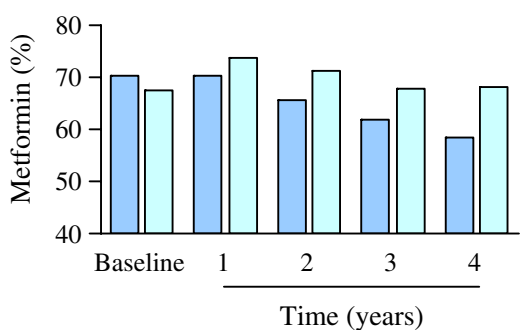


above, the prevalence of complications was high. Among the VADT patients, along with diabetic neuropathy, retinopathy was present in $62 \%$ of the diabetic patients and $40 \%$ of them had already experienced a CV event. No doubt this was a high-risk diabetic population. In agreement with the high rate of complications, patients had poor glycaemic control at baseline, as indicated by an average $\mathrm{HbA}_{1 \mathrm{c}}$ of $9.4 \%$, not a surprising finding in patients with an average disease duration of 11.4 years. The question is how much the previous history of diabetes may have influenced intervention outcome. One way of addressing such a hypothesis is to reconstruct the history of the disease. Given the duration of diabetes in these individuals, one may assume its course to be much like the one described by the UKPDS, graphically depicted in Fig. 2. It is likely that this individual had a high $\mathrm{HbA}_{1 \mathrm{c}}$ level at the time of diagnosis and also that he or she was started on dietary therapy and/or some oral hypoglycaemic agents that improved, but did not normalise, glycaemic control. Nonetheless, over the time the patient's glycaemic control worsened progressively to reach, 11.4 years later on, a $\mathrm{HbA}_{1 \mathrm{c}}$ level of $9.4 \%$, when he/ she was recruited to participate in the VADT. Intensive therapy implemented in the trial resulted in a rapid lowering of plasma glucose levels and subsequent maintenance of $\mathrm{HbA}_{1 \mathrm{c}}$ close to the ideal target. It is, however, readily apparent that this time course is far from the ideal, i.e. the early achievement and maintenance of near-normal glycaemia from the time of diagnosis. The difference between the ideal and the actual time course of glycaemic control represents a time period (Fig. 2) that may have had some impact on the effect of subsequent tight glycaemic control. First of all, preceding longstanding hyperglycaemia accounts for the high rate of complications at baseline. Second, it may have generated a 'bad glycaemic legacy'. The concept of glycaemic legacy has been proposed based on the long-term results of the DCCT-Epidemiology of Diabetes Interventions and Complications (EDIC) study $[28,29]$. Upon completion of the active trial, type 1 diabetic patients originally on conventional treatment were encouraged to switch to intensive treatment, whereas in those intensively treated, a less tight control evolved. As a consequence of all this, the original difference in $\mathrm{HbA}_{1 \mathrm{c}}$ ( $7 \%$ vs $9 \%$ ) was progressively lost so that, 3 years later, glycaemic control was similar between the two groups $\left(\mathrm{HbA}_{1 \mathrm{c}} \sim 8 \%\right)$. In spite of this, the risk reduction for microvascular complications recorded at the end of the trial was fully retained [29]. Even more striking was the risk reduction for $\mathrm{CV}$ disease (any $\mathrm{CV}$ event $-42 \%, 95 \% \mathrm{CI}$ $9-63 \%, p=0.02$ ) and for non-fatal myocardial infarction, stroke or death from CV disease $(-57 \%, 95 \%$ CI $12-7 \%$, $p=0.02$ ) recorded during the 17 year follow-up period [30]. A legacy effect has now also been confirmed for type 2 diabetes [30]. In the UKPDS 10 year post-trial follow-up, patients originally randomised to receive intensive treatment, not only still showed significant reductions in the rates of diabetesrelated endpoints and microvascular complications, but were also at a greatly reduced risk of myocardial infarction (RR reduction $15 \%, p=0.0014$ ) and all-cause mortality (RR reduction $13 \%, p=0.007)$. Interestingly, no such legacy seems to be associated with early blood pressure control, as in the UKPDS the positive effect of intensive treatment on microand macrovascular complications was lost at follow-up [31].

Together, these results suggest that, at least within the timeframe of the intensive treatment period of the most recent trials, there is less opportunity to influence the development and/or progression of complications in individuals with longstanding diabetes. Conversely, in both type 1 [28, 29] and type 2 [30] diabetic patients, early strict glycaemic control generates a legacy that may confer protection against, or delay, long-term diabetic complications. Data in support
Fig. 2 Hypothetical representation of the natural history of the diabetic patients recruited in the VADT. The upper dotted line represents the time course of $\mathrm{HbA}_{1 \mathrm{c}}$ estimated on the basis of the average glucose profile described by the UKPDS [2]. The lower dotted line represents the ideal time course of glycemic control. The solid line represents the time course of $\mathrm{HbA}_{1 \mathrm{c}}$ in the VADT [8]

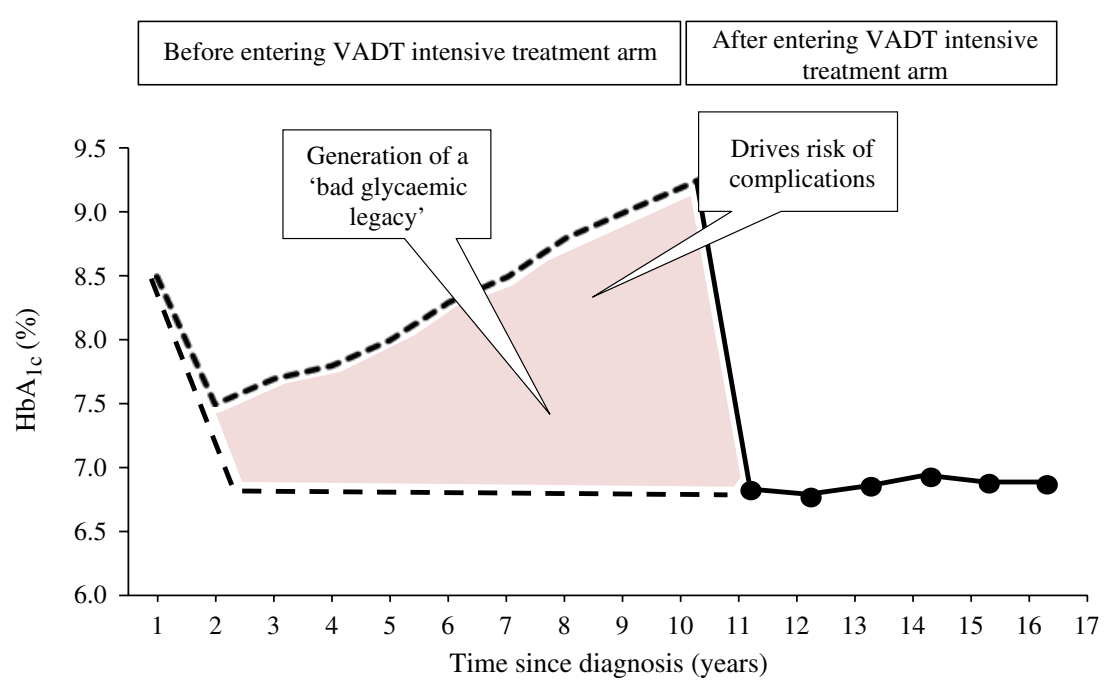


of this view can also be derived from the ACCORD trial [6]. In spite of an increased risk of mortality in intensively treated patients, per group analysis indicates a significant HR reduction with respect to primary $\mathrm{CV}$ endpoint in diabetic patients with no previous $\mathrm{CV}$ events $(p=0.04)$ and in those with a $\mathrm{HbA}_{1 \mathrm{c}}$ level of $\leq 8 \%(p=0.03)$. Moreover, the VADT investigators reported that the $\mathrm{HR}$ for $\mathrm{CV}$ disease owing to intensive glycaemic control varies as a function of disease duration: it is much reduced in diabetic patients with short disease duration, and this reduction becomes smaller with longer disease duration, to actually worsen in individuals with longstanding diabetes [32].

\section{Micro- and macrovascular complications-a common soil?}

Although the results of the UKPDS post-trial results were felt reassuring, the recent published results of the VADT [8], ACCORD [6], and ADVANCE [7] have left a feeling of uneasiness on the light of limited effect not just on $\mathrm{CV}$ event, a very debated issue, but also because of poor microvascular complication outcome. These findings, however, may be less striking if we avoid looking at micro- and macrovascular complications as two separate entities. Literature is now available to show that retinopathy predicts CV mortality in type 2 diabetes [33]. A cross-sectional association between coronary artery calcium (CAC) and proliferative diabetic retinopathy has been reported in a VADT subsample of diabetic patients [34]. Of note, the association remained statistically significant even after adjustment for multiple CV risk factors. Individuals with proliferative diabetic retinopathy had a sixfold higher risk of having a CAC score of $>400$. This sort of association is not surprising given the many common pathophysiological mechanisms, including inflammation, hypercoagulability state and endothelial dysfunction [35]. The possible contribution of both traditional and non-traditional CV risk factors to the development and progression of microvascular complications has already been mentioned above. Finally, hyperglycaemia and associated metabolic alterations may well represent a single unifying process producing diabetic complications through activation of oxidative stress [36]. It is therefore not surprising that in patients with longstanding diabetes and consolidated micro- and macrovascular damage, 6 years of intensive glycaemic control may not result in any apparent beneficial effect.

\section{Conclusion and perspectives}

At the press conference that followed the presentation of the VADT on the occasion of the 68th Scientific Sessions of the
ADA, William C. Duckworth, the VADT principal investigator, commented: If you go into a population that already has multiple risk factors - or prior cardiovascular disease-and longstanding poor glucose control, you cannot expect benefits from glucose control in the short term. You can't expect miracles.' [37]. But it is not a miracle that we look for, but, rather, rational approaches for effective treatment and prevention of diabetic complications.

A large number of diabetic patients still have poor glycaemic control [9]. Their $\mathrm{HbA}_{1 \mathrm{c}}$ must be lowered but we should be very careful in deciding how low the target should be and how to reach it. Individualisation of the glycaemic target is definitely needed in people with existing micro- and macrovascular complications. Reduction of the risk of severe hypoglycaemia is desirable given the association with CV events. Risk-to-benefit assessment of available glucose-lowering agents should be performed so that an appropriate treatment strategy is selected. An improvement in $\mathrm{HbA}_{1 \mathrm{c}}$ may be attained with slow but progressive decline. In both the VADT [8] and ACCORD [6] trials, $\mathrm{HbA}_{1 \mathrm{c}}$ declined very quickly, and such a rapid improvement has been associated, in controlled studies, with initial worsening of existing diabetic complications $[38,39]$. Interestingly, a much smoother reduction in $\mathrm{HbA}_{1 \mathrm{c}}$ was obtained in the ADVANCE trial [7]. Although the real implications of a rapid achievement of glycaemic control may not be fully apparent, the risk of hypoglycaemia is likely to be greater if a more aggressive approach is used. The use of statins and effective anti-hypertensive treatment should be initiated irrespective of previous CV events in all diabetic patients. However, a true shift in treatment strategy for type 2 diabetes will require the implementation of appropriate treatment at the time of the diagnosis [40], if not earlier on. The probability of there being existing diabetic complications is lower at diagnosis, allowing effective protection. The 'metabolic legacy' is short in duration and hence easier to be modified. In these patients, therefore, targeting normoglycaemia is not just feasible but mandatory. One possible strategy would be to avoid agents that may expose the patient to an unnecessary risk of hypoglycaemia. From this point of view, taking into consideration the pathophysiological basis of hyperglycaemia may provide guidance for the selection of medications. In all cases, however, an uncompromised therapeutic insistence should be adopted [41], including the treatment of all CV risk factors.

Ideally, diabetes prevention should be desirable, but while we wait for effective and feasible preventative procedures, efforts should be made to treat-to-target all newly diagnosed diabetic patients. Finally, we should all remember and emphasise to the entire medical community that no form of mild diabetes exists, and no excuse exists to postpone appropriate and effective treatment. 
Acknowledgements I would like to thank R. Miccoli and G. Penno at the University of Pisa (Pisa, Italy) for continuous support and critical suggestions during the preparation of my commentary to the VADT on the occasion of the 44th Annual Meeting of the European Association for the Study of Diabetes, Rome, 8-11 September 2008, as well as during the preparation of this manuscript. A special thank you to R. Bonadonna (University of Verona, Verona, Italy), who provided useful suggestions following critical reading of the manuscript.

Duality of interest The author declares the following potential duality of interest: participation in advisory panels for Novartis Pharmaceuticals, Merck, Roche Pharmaceuticals, Roche Diagnostics, Pfizer, Eli Lilly, Amylin Pharmaceuticals, Mannkind, Boehringer Ingelheim, Bristol-Myers Squibb, AstraZeneca, GlaxoSmithKline, sanofi-aventis and Takeda Pharmaceuticals; research support received from Merck, sanofi-aventis and Takeda Pharmaceuticals.

\section{References}

1. University Group Diabetes Program (UGDP) (1970) A study of the effects of hypoglycemic agents on vascular complications in patients with adult-onset diabetes. Diabetes 19(Suppl 2):747-830

2. UK Prospective Diabetes Study (UKPDS) Group (1998) Intensive blood-glucose control with sulphonylureas or insulin compared with conventional treatment and risk of complications in patients with type 2 diabetes (UKPDS 33). Lancet 352:837-853

3. Genuth S, Eastman R, Kahn R, American Diabetes Association et al (2003) Implications of the United Kingdom prospective diabetes study. Diabetes Care 26:S28-S32

4. Ohkubo Y, Kishikawa H, Araki E et al (1995) Intensive insulin therapy prevents the progression of diabetic microvascular complications in Japanese patients with non-insulin-dependent diabetes mellitus: a randomized prospective 6-year study. Diabetes Res Clin Pract 28:103-117

5. Dormandy JA, Charbonnel B, Eckland DJ (2005) Secondary prevention of macrovascular events in patients with type 2 diabetes in the PROactive Study (PROspective pioglitAzone Clinical Trial In macroVascular Events): a randomised controlled trial. Lancet 366:1279-1289

6. The Action to Control Cardiovascular Risk in Diabetes Study Group (2008) Effects of intensive glucose lowering in type 2 diabetes. N Engl J Med 358:2545-2559

7. The ADVANCE Collaborative Group (2008) Intensive blood glucose control and vascular outcomes in patients with type 2 diabetes. N Engl J Med 358:2560-2572

8. Duckworth W, Abraira C, Moritz T et al (2009) Glucose control and vascular complications in veterans with type 2 diabetes. $\mathrm{N}$ Engl J Med 360:129-139

9. Ong KL, Cheung BM, Wong LY, Wat NM, Tan KC, Lam KS (2008) Prevalence, treatment, and control of diagnosed diabetes in the U.S. National Health and Nutrition Examination Survey 1999 2004. Ann Epidemiol 18:222-229

10. Dale AC, Vatten LJ, Nilsen TI, Midthjell K, Wiseth R (2008) Secular decline in mortality from coronary heart disease in adults with diabetes mellitus: cohort study. BMJ 337:99-102

11. The Diabetes Control and Complications Trial Research Group (1993) The effect of intensive therapy of diabetes on the development and progression of long-term complications in insulin-dependent diabetes mellitus. N Engl J Med 329:977-986

12. Klein R, Knudtson MD, Lee KE, Gangnon R, Klein BE (2008) The Wisconsin Epidemiologic Study of Diabetic Retinopathy: XXII the twenty-five-year progression of retinopathy in persons with type 1 diabetes. Ophthalmology 115:1859-1868
13. Girach A, Manner D, Porta M (2006) Diabetic microvascular complications: can patients at risk be identified? A review. Int J Clin Pract 60:1471-1483

14. Stratton IM, Cull CA, Adler AI, Matthews DR, Neil HA, Holman RR (2006) Additive effects of glycaemia and blood pressure exposure on risk of complications in type 2 diabetes: a prospective observational study (UKPDS 75). Diabetologia 49:1761-1769

15. Gaede P, Vedel P, Larsen N, Jensen GV, Parving HH, Pedersen O (2003) Multifactorial intervention and cardiovascular disease in patients with type 2 diabetes. N Engl J Med 348:383-393

16. Kilo C, Miller JP, Williamson JR (1980) The Achilles heel of the University Group Diabetes Program. JAMA 243:450-457

17. UK Prospective Diabetes Study (UKPDS) (1998) Group Effect of intensive blood-glucose control with metformin on complications in overweight patients with type 2 diabetes (UKPDS 34). UK Prospective Diabetes Study (UKPDS) Group. Lancet 352:854865

18. Evans JM, Ogston SA, Emslie-Smith A, Morris AD (2006) Risk of mortality and adverse cardiovascular outcomes in type 2 diabetes: a comparison of patients treated with sulfonylureas and metformin. Diabetologia 49:930-936

19. Kronmal RA, Barzilay JI, Smith NL et al (2006) Mortality in pharmacologically treated older adults with diabetes: the Cardiovascular Health Study, 1989-2001. PLoS Med 3:e400

20. Anselmino M, Ohrvik J, Malmberg K, Standl E, Rydén L, Euro Heart Survey Investigators (2008) Glucose lowering treatment in patients with coronary artery disease is prognostically important not only in established but also in newly detected diabetes mellitus: a report from the Euro Heart Survey on Diabetes and the Heart. Eur Heart J 29:177-184

21. Abraira C, Duckworth WC, Moritz T et al (2008) Glycaemic separation and risk factor control in the Veterans Affairs Diabetes Trial: an interim report. Diabetes Obes Metab 11:150-156

22. Byington RP for the ACCORD Study Group (2008) Relationship between post-randomization hypoglycaemia and ACCORD mortality outcomes. Available from http://webcasts.prous.com/netadmin/ webcast_viewer/Preview.aspx?type $=0 \&$ lid $=3753$, accessed 20 March 2009

23. Hilsted J (1993) Cardiovascular changes during hypoglycaemia. Clin Physiol 13:1-10

24. Avogaro A, Vigili de Kreutzenberg S, Negut C, Tiengo A, Scognamiglio R (2004) Diabetic cardiomyopathy: a metabolic perspective. Am J Cardiol 93:13A-16A

25. Landstedt-Hallin L, Englund A, Adamson U, Lins PE (1999) Increased QT dispersion during hypoglycaemia in patients with type 2 diabetes mellitus. J Intern Med 246:299-307

26. Monnier L, Mas E, Ginet C et al (2006) Activation of oxidative stress by acute glucose fluctuations compared with sustained chronic hyperglycemia in patients with type 2 diabetes. JAMA 295:1681-1687

27. Vinik AI, Ziegler D (2007) Diabetic cardiovascular autonomic neuropathy. Circulation 115:387-397

28. White NH, Sun W, Cleary PA, Danis RP et al (2008) Prolonged effect of intensive therapy on the risk of retinopathy complications in patients with type 1 diabetes mellitus: 10 years after the Diabetes Control and Complications Trial. Arch Ophthalmol 126:17071715

29. Nathan DM, Cleary PA, Backlund JY, Diabetes Control and Complications Trial/Epidemiology of Diabetes Interventions and Complications (DCCT/EDIC) Study Research Group et al (2005) Intensive diabetes treatment and cardiovascular disease in patients with type 1 diabetes. N Engl J Med 353:2643-2653

30. Holman RR, Paul SK, Bethel MA, Matthews DR, Neil HA (2008) 10 -year follow-up of intensive glucose control in type 2 diabetes. N Engl J Med 359:1577-1589 
31. Holman RR, Paul SK, Bethel MA, Neil HA, Matthews DR (2008) Long-term follow-up after tight control of blood pressure in type 2 diabetes. N Engl J Med 359:1565-1576

32. Duckworth W (2008) VADT: results. Available from http:// webcasts.prous.com/netadmin/webcast_viewer/Preview.aspx? type $=0 \&$ lid $=3853$, accessed 20 March 2009

33. Juutilainen A, Lehto S, Rönnemaa T, Pyörälä K, Laakso M (2007) Retinopathy predicts cardiovascular mortality in type 2 diabetic men and women. Diabetes Care 30:292-299

34. Reaven PD, Emanuele N, Moritz T, Veterans Affairs Diabetes Trial et al (2008) Proliferative diabetic retinopathy in type 2 diabetes is related to coronary artery calcium in the Veterans Affairs Diabetes Trial (VADT). Diabetes Care 31:952-957

35. Moreno PR, Fuster V (2004) New aspects in the pathogenesis of diabetic atherothrombosis. J Am Coll Cardiol 44:22932300

36. Brownlee M (2005) The pathobiology of diabetic complications: a unifying mechanism. Diabetes 54:1615-1625
37. http://www.diabetes.org/for-media/pr-intense-blood-glucose-controlyields-no-significant-effect-on-cvd-reduction.jsp, accessed on 2 September 2006

38. The DCCT Research Group (1998) Early worsening of diabetic retinopathy in the Diabetes Control and Complications Trial. Arch Ophthalmol 116:874-886

39. Lauritzen T, Frost-Larsen K, Larsen HW, Deckert T (1983) Effect of 1 year of near-normal blood glucose levels on retinopathy in insulin-dependent diabetics. Lancet 1:200-204

40. Del Prato S, Felton AM, Munro N, Nesto R, Zimmet P, Zinman B, Global Partnership for Effective Diabetes Management (2005) Improving glucose management: ten steps to get more patients with type 2 diabetes to glycaemic goal. Int J Clin Pract 59:1345-1355

41. Lebovitz HE, Austin MM, Blonde L, ACE/AACE Diabetes Recommendations Implementation Writing Committee et al (2006) $\mathrm{ACE} / \mathrm{AACE}$ consensus conference on the implementation of outpatient management of diabetes mellitus: consensus conference recommendations. Endocr Pract 12(Suppl 1):6-12 